\title{
Monitoring A Large Construction Site Using Wireless Sensor Networks
}

\author{
Sarfraz Nawaz \\ Computer Laboratory \\ University of Cambridge \\ sarfraz.nawaz@cl.cam.ac.uk \\ Paul Fidler \\ Department of Engineering \\ University of Cambridge \\ praf1@cam.ac.uk
}

\author{
Xiaomin Xu \\ Department of Engineering \\ University of Cambridge \\ xx787@cam.ac.uk \\ Kenichi Soga \\ Department of Engineering \\ University of Cambridge \\ ks207@cam.ac.uk
}

\author{
David Rodenas-Herráiz \\ Computer Laboratory \\ University of Cambridge \\ dr424@cl.cam.ac.uk \\ Cecilia Mascolo \\ Computer Laboratory \\ University of Cambridge \\ cecilia.mascolo@cl.cam.ac.uk
}

\begin{abstract}
Despite the significant advances made by wireless sensor network research, deployments of such networks in real application environments are fraught with significant difficulties and challenges that include robust topology design, network diagnostics and maintenance. Based on our experience of a six-month-long wireless sensor network deployment in a large construction site, we highlight these challenges and argue the need for new tools and enhancements to current protocols to address these challenges.
\end{abstract}

\section{INTRODUCTION}

Wireless Sensor Networks (WSN) have emerged as a promising technology for large-scale sensing in various fields including agriculture, environmental and infrastructure monitoring over the last decade. This has led to the development of a suite of hardware platforms and software frameworks that include advanced communication protocols. While the initial focus of sensor network research has been energy efficiency, recent efforts have led to a standardized set of protocols that are not only energy efficient but which also offer benefits of interoperability and ease of connectivity to the wider Internet.

Despite the significant advances in sensor network design, real deployments are fraught with several challenges that make it difficult to quickly and efficiently deploy a wireless sensor network in a real application environment. These challenges include network topology design, diagnostics and maintenance of the network. In this paper, we present the difficulties and challenges that we experienced during sixmonth-long infrastructure monitoring deployment using wireless sensor networks on a large construction site. A construction site presents a challenging environment with cluttered spaces, limited and expensive access and a physical layout

Permission to make digital or hard copies of all or part of this work for personal or classroom use is granted without fee provided that copies are not made or distributed for profit or commercial advantage and that copies bear this notice and the full citation on the first page. Copyrights for components of this work owned by others than ACM must be honored. Abstracting with credit is permitted. To copy otherwise, or republish, to post on servers or to redistribute to lists, requires prior specific permission and/or a fee. Request permissions from Permissions@ acm.org.

RealWSN'15, November 1, 2015, Seoul, South Korea.

(C) 2015 ACM. ISBN 978-1-4503-3840-0/15/11 ...\$15.00.

DOI: http://dx.doi.org/10.1145/2820990.2820997. that changes over the time. This makes it difficult to predesign a suitable network topology and requires fast on-site diagnostics of any network problems that may arise during and after the deployment. Our experience with WSN deployments on construction sites suggests that there is a strong need for a streamlined strategy, enhancements to existing network protocols and a set of tools that can be used to design robust network topologies with minimum on-site measurements and diagnose any problems or issues that the network may be experiencing while on site. We describe the monitoring requirements and construction site layout in Section (2), and the wireless sensor network system used for this deployment in Section (3). Section (4) discusses the network dynamics and issues that we experienced during the lifetime of this network. Section (5) summarises the challenges that we feel need to be addressed for wireless sensor networks to become a wide spread monitoring technology.

\section{CONSTRUCTION SITE}

The site for the WSN deployment is an excavation for a new Crossrail station at Paddington, London. This excavation takes the form of an underground box that is $260 \mathrm{~m}$ long, $25 \mathrm{~m}$ wide and $23 \mathrm{~m}$ deep. It is made up of 165 rectangular diaphragm wall panels, 51 plunge columns and 100 giant hydraulic props as shown in Fig. (1) and Fig. (2). Each of the wall panels is $3.3 \mathrm{~m} \times 1.2 \mathrm{~m} \times 40 \mathrm{~m}$. Construction started on this site in October 2011 and is due to be completed during 2017.

The main aim of the WSN deployment was to monitor deformation of three diaphragm wall panels on one of the corners of this underground box during excavation. It was anticipated that the corner would produce a stiffening effect on the station box, resulting in a breakdown of plane strain conditions and a reduction in lateral panel movement. Wireless tilt and displacement sensors were installed to measure inclination, angular distortion and relative displacement of these corner panels at two different depths. These measurements can potentially offer some insights on the real performance of a box corner during large deep excavation. The WSN gateway was positioned outside the underground box, as it requires a power supply and good 3G signal coverage. Several relay nodes were also attached to the diaphragm wall panels and plunge columns. 


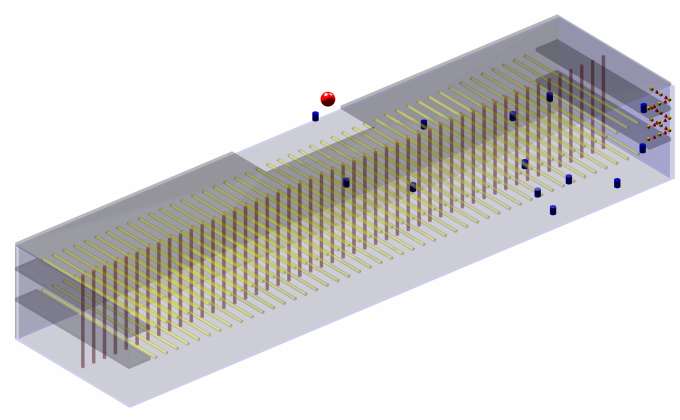

Figure 1: 3D model of Paddington station main box and WSN layout.

\section{WIRELESS SENSOR NETWORK}

The tilt and displacement sensors used in the deployment were obtained from Wisen Innovation. These sensors are commercially available and designed for use on construction sites and are packaged in robust metal housings. Internally the devices are based on the AVR ATmega1281 processor and the IEEE 802.15.4-compliant AT86RF231 radio. Other radio technologies, such as $\mathrm{Wi}-\mathrm{Fi}$, were considered unsuitable mainly due to their high power consumption. Fifteen sensors measured displacement using an LPDT while twelve measured tilt using Murata SCA100T MEMS inclinometers. For the relay nodes Dresden Elektronik deRFmega128 modules were used. These modules were packaged in an off-theshelf plastic housing, initially with no external antennas in order to maintain the ingress protection rating. Thirteen relays were used in total.

The gateway used a Memsic Iris mote acting as the root node and border router. This was attached to a Memsic MIB520 Gateway with data transferred over a USB connection and logged using a Raspberry Pi single board computer. Internet connectivity was provided by a $3 \mathrm{G}$ USB modem, and there was provision to reboot the Raspberry Pi remotely using an SMS-controlled switch. The use of the MIB520 gateway board allowed for resetting or reprogramming the Iris remotely if required.

The application software running on the wireless sensor devices was developed in Contiki OS [1]. Contiki OS was relatively easy to port to the Wisen hardware as it is internally similar to the Memsic Iris for which there was already a Contiki OS port. Nodes use Contiki's standards-based IPv6 stack (6LoWPAN/RPL) for link-local addressing and routing, and ContikiMAC at MAC layer for low-power operation. As is typical in multi-hop networks, the expected transmission count (ETX) metric [2] is used for link quality estimation. Each node was initially programmed to send link-local information periodically to assist in identifying and diagnosing potential network failures. This information included network-layer statistics, the current RPL parent node, and the neighbour table containing neighbouring nodes. Extensive testing and calibration was undertaken in the laboratory to ensure that the system functioned as expected.

\section{NETWORK DYNAMICS}

The layout of the wireless sensor network at the Paddington excavation is shown in Fig. (3b). The WSN installation took place over two days in February 2014. Sensor data mes-

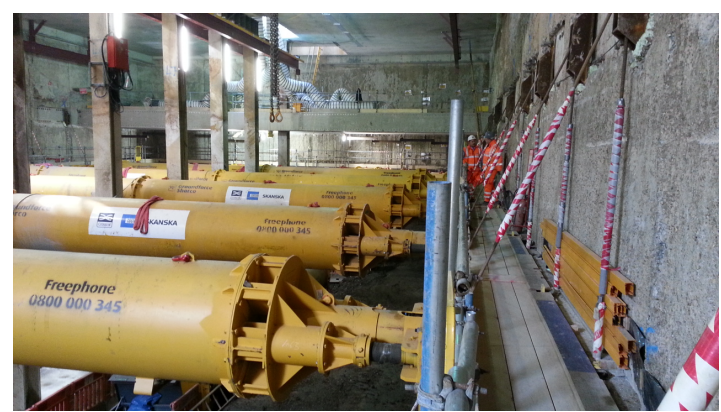

Figure 2: Inside the station main box with sensors installed in far corner.

sages are sent from each node at fifteen minutes intervals. In addition, both the relays and the sensor nodes were also configured to send two different diagnostic messages containing information about network connectivity every fifteen minutes. The transmissions were not synchronized. The message delivery ratio for every node was obtained as the number of messages successfully delivered to the gateway with respect to the total number of expected data transmissions.

Fig. (4) shows the data message delivery ratio computed for two individual LPDT sensors (labelled 4 and 5) during the deployment. The network experienced continuous connectivity problems that resulted in data message delivery ratios of below $10 \%$ in the two first months after deployment. Investigation were undertaken to diagnose the cause of the low data message delivery ratio. Diagnostics were limited to analysis of those network connectivity diagnostic messages that successfully reached the gateway. All received messages were time stamped with their time of arrival at the gateway. It can be observed from Fig. (4) that the delivery ratio for the diagnostic information was also below $10 \%$. The time difference between the reception of consecutive messages was irregular, rather than the expected fifteen minutes. For these reasons it was very difficult to determine the root causes of such a high message loss.

Although insufficient, the analysis of diagnostic data did provide some insight into the actual network behaviour. Fig. (3b) shows the network topology constructed from the diagnostic messages sent by all nodes in the WSN. Interestingly, it shows that sensor nodes were routing messages via a single far-off relay which was located on the opposite side of the station box in close proximity to the gateway, rather than using nearby relay nodes to forward messages. It was observed that the sensors did not forward messages via the relay nodes placed further along the concrete retaining wall. A potential reason for this was thought to be the Wall Antenna effect [3] which would have limited radio propagation in this direction. This prompted the installation of two additional relay nodes in an attempt to increase path diversity and reduce the routing overhead at the far-off relay node. Fig. (3c) shows the new network topology after the addition of relay nodes shown as magenta squares. Due to the lack of detailed diagnostic information, the positions for the new relay nodes were chosen intuitively based on experience gained from previous deployments.

With the installation of the two additional relays, an improvement in data message delivery ratio for all sensor nodes (up to three times more) was observed. Unfortunately, this 


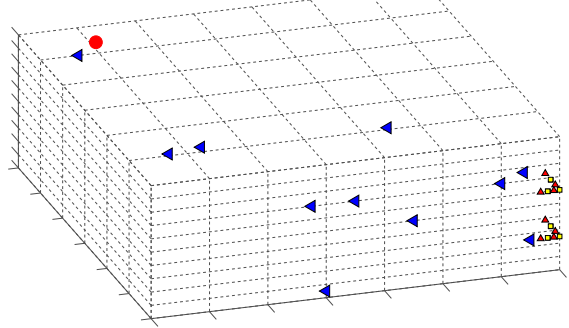

(a)

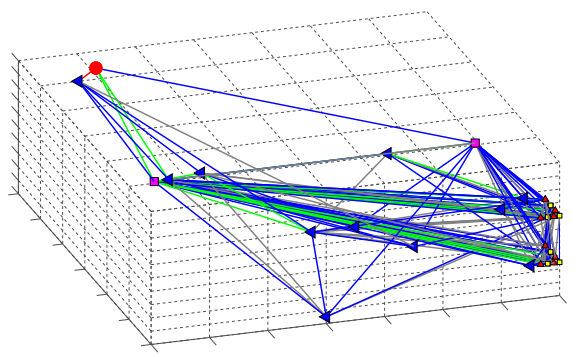

(c)

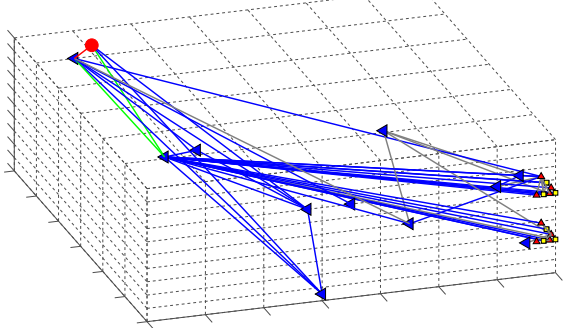

(b)

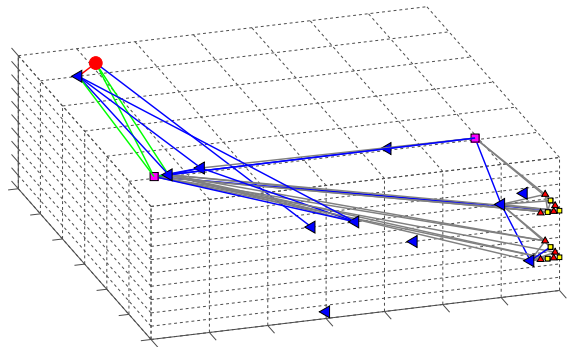

(d)

Figure 3: Network layout: (a) Initial network layout; (b) Initial topology; (c) Two relay nodes were added to introduce path diversity; (d) Final network topology at the end of six months. Link color represents the average number of connections made to the gateway per day during the 5-day period. Grey line indicates one-two connections; blue line, between two and 20 connections; green line, between 20 and 200 connections; and red line, more than 200 connections.

improvement only lasted for around twenty days, after which the message delivery ratios dropped again. Fig. (3d) shows the network topology constructed from the limited amount of diagnostic data reaching the gateway. It shows that sensor nodes now had limited connectivity to the relay node closest to the gateway. In a network suffering from high message loss, sending diagnostic information to the gateway does not provide any detailed insight into the cause of these losses. Therefore, all the nodes in the network were reprogrammed with new application software. This new application stores diagnostic data in local non-volatile (flash) memory rather than sending this information via lossy links. This stored data may be retrieved during repeated site visits.

\section{DISCUSSION}

\subsection{Network Topology Design}

A major factor in network topology design is the placement of relay nodes so that all the nodes can form a robust network topology and the gateway can receive data from sensor nodes effectively with minimum message loss. In general, the location of the sensor nodes is dependent on the monitoring task. At our Paddington site, sensor nodes were placed to monitor the relative movements of separately-cast concrete wall panels at the corner of the station box. The position of the relay nodes was not so constrained. In a relatively dense network with many relays, the RPL routing protocol implemented by Contiki OS will automatically discover a network topology [4] providing sensor nodes with a variety of routing paths. However, deploying many redundant relays is not realistic in many real applications including construction sites due to difficulties with access and the need to keep system costs low. On the other hand, sparse networks can lead to problems that we experienced on our deployment site. Therefore, there is a strong need for effective and practical topology design approaches [5] for this type of site environment. Special consideration should also be given to routing protocols [6] as well as alternative transmission techniques, such as band diversity [7] or directional antenna, which seek to improve the delivery ratio even though they may have a power consumption penalty.

\subsection{Diagnostics}

The need for data messages to actually reach the gateway node in order to be logged and therefore be available for diagnostic purposes proved to be a major limitation. Without a reliable connection to each node it is difficult to diagnose the problems that caused the unreliability. In our installation we had to resort to looking carefully at the few successfully transmitted network connectivity diagnostic messages transmitted by neighbouring nodes to glean any useful information about those nodes from which we did not receive any data. A portable diagnostic tool would have enabled us to identify which nodes were transmitting, and which nodes they had selected as their RPL parent node even if messages did not get as far as the gateway. A small hand-held tablet-like device would be convenient for construction site diagnostics provided site safety issues were considered.

\subsection{Maintenance}

Construction sites present particular problems for WSNs as the layout of a site changes over time. New walls, slabs and columns are built, new supports may be added, whilst others are removed, and vehicles and plant movements will constantly change the nature of a site in ways that may affect the functioning of a WSN. Problems may occur that 


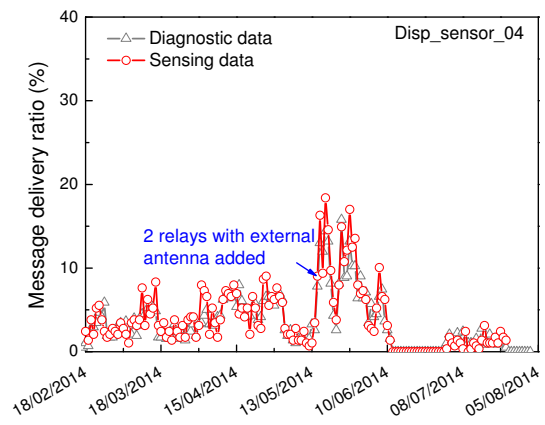

(a)

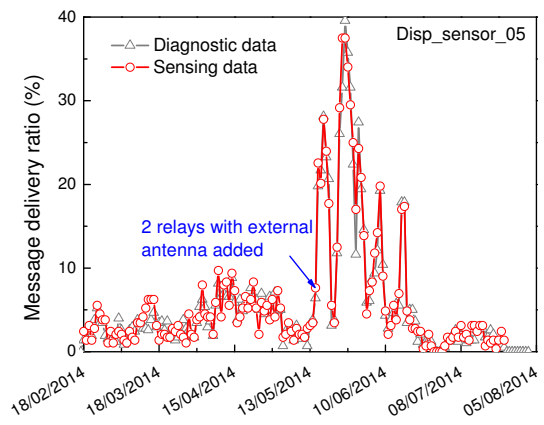

(b)

Figure 4: Data and diagnostic message delivery ratio at the gateway from two displacement sensors attached to diaphragm panels in the corner of the box.

would simply not happen in a laboratory environment [8]. For example, at Paddington Station one of the relay nodes was removed by a site operative as it was obstructing work. It was not replaced afterwards. When problems such as this arise, it is important to be able to diagnose the problem quickly, ideally remotely, and then prepare a fix off site. Site access is often limited and the associated site-access costs may be too high to allow for repeated on-site visits.

\section{CONCLUSION}

Despite the development of the core communications protocols necessary to enable wireless sensor networking, deployments on real-world sites can still be problematic. In the absence of suitable diagnostic data available at the gateway node it can be difficult on a large site to quickly diagnose and fix issues such as poor point-to-point link quality caused by sub-optimal relay placement.

Support tools to assist with the rapid deployment, diagnosis and maintenance of such WSNs are required. Whilst this is especially true of construction sites, where speed of deployment is an important consideration due to limited or expensive access, such tools would also be of benefit to other deployment scenarios outside the laboratory.

\section{ACKNOWLEDGEMENTS}

This research has been funded by the EPSRC Innovation and Knowledge Centre for Smart Infrastructure and Construction project (EP/K000314/1). We would like to thank Costain-Skanska Joint Venture (CSJV) and our industrial partner Crossrail for allowing access and instrumentation of the Paddington site. We would also like to thank Dr Munenori Shibata from Japan Railway Technical Research Institute for his assistance with network deployment. Data supporting this paper is available from https://www.repository.cam.ac.uk/handle/1810/250538

\section{REFERENCES}

[1] Adam Dunkels, Björn Grönvall, and Thiemo Voigt. Contiki - a lightweight and flexible operating system for tiny networked sensors. In Proceedings of the 29th Annual IEEE International Conference on Local Computer Networks, LCN 2004, pages 455-462, Tampa, Florida, USA, November 2004. IEEE Computer Society.
[2] Douglas S. J. De Couto, Daniel Aguayo, John Bicket, and Robert Morris. A high-throughput path metric for multi-hop wireless routing. In Proceedings of the 9th Annual International Conference on Mobile Computing and Networking, MobiCom '03, pages 134-146. ACM, 2003.

[3] Neil A. Hoult, Yan Wu, Ian J. Wassell, Peter J. Bennett, Kenichi Soga, Campbell R. Middleton. Challenges in Wireless Sensor Network Installation: Radio Wave Propagation. In Proceedings of the 4 th International Conference on Structural Health Monitoring of Intelligent Infrastructure, Zurich, Switzerland, July 2009.

[4] Nicolas Tsiftes, Joakim Eriksson, and Adam Dunkels. Low-power Wireless IPv6 Routing with ContikiRPL. In Proceedings of the 9th ACM/IEEE International Conference on Information Processing in Sensor Networks, IPSN '10, pages 406-407, Stockholm, Sweden, 2010. ACM.

[5] Yin Chen and Andreas Terzis. On the Implications of the Log-normal Path Loss Model: An Efficient Method to Deploy and Move Sensor Motes. In Proceedings of the 9th ACM Conference on Embedded Networked Sensor Systems, SenSys 2011, pages 26-39, Seattle, Washington, USA, November 2011. ACM.

[6] Tomonori Nagayama, Parya Moinzadeh, Kirill A. Mechitov, Mitsushi Ushita, Noritoshi Makihata, Masataka Leiri, Gul Agha, Billie F. Spencer, Jr., Yozo Fujino, and Ju Won Seo. Reliable multi-hop communication for structural health monitoring. Smart Structures and Systems, 6(5-6):481-504, 2010.

[7] Branislav Kusy, David Abbott, Christian Richter, Cong Huynh, Mikhail Afanasyev, Wen Hu, Michael Brünig, Diethelm Ostry, and Raja Jurdak. Radio diversity for reliable communication in sensor networks. $A C M$ Transactions on Sensor Networks, 10(2):32:1-32:29, January 2014.

[8] Ian F. Akyildiz and Mehmet Can Vuran. Factors Influencing WSN Design. In Wireless Sensor Networks, Ian F. Akyildiz Series in Communications and Networking, chapter 3, pages 37-51. John Wiley \& Sons, Ltd, 2010 